This article is licensed under the Creative Commons Attribution-NonCommercial 4.0 International License (CC BY-NC) (http://www.karger.com/Services/OpenAccessLicense). Usage and distribution for commercial purposes requires written permission.

\title{
Herpes Simplex Virus Type 2 Radiculomyelitis Disguised as Conversion Disorder
}

\author{
Lisa B.E. Shields Mohammad S. Alsorogi \\ Norton Neuroscience Institute, Norton Healthcare, Louisville, KY, USA
}

\section{Keywords}

Neurology · Herpes simplex virus · Radiculomyelitis · Conversion disorder · Polymerase chain reaction

\begin{abstract}
Herpes simplex virus type 2 (HSV-2) is the most common cause of genital herpes with a seroprevalence of $20-30 \%$ in developed countries and $80 \%$ worldwide. In addition to neonatal encephalitis and meningitis, HSV-2 is associated with radiculomyelitis marked by pain, paresis, sphincter disturbances, sensory loss, or ascending necrotizing myelitis. We report the case of a patient with a lengthy psychiatric history who presented with lower extremity pain and weakness. Cervical, thoracic, and lumbar MRI scans with and without gadolinium contrast revealed no significant stenosis, neural compression, or other abnormal findings, and the brain MRI with and without gadolinium contrast was normal. The initial diagnosis was conversion disorder due to myriad psychological stressors. Polymerase chain reaction (PCR) of CSF detected HSV-2 and a lymphocytic pleocytosis, and the diagnosis of radiculomyelitis was confirmed. She was treated with i.v. acyclovir for 3 weeks followed by valacyclovir. The patient attained no improvement of her symptoms within 8 months; however, she reported decreased pain and improved strength of the lower extremities by 17 months. Neurologists should be aware of the
\end{abstract}




\section{Case Reports in Neurology}

Case Rep Neurol 2019;11:117-123

DOI: $10.1159 / 000499701$

2019 The Author(s). Published by S. Karger AG, Base www.karger.com/crn

Shields and Alsorogi: Herpes Simplex Virus Type 2 Radiculomyelitis Disguised as Conversion Disorder

association between HSV-2 and radiculomyelitis, particularly in the setting of a patient with psychiatric comorbidities. Recognition of HSV-2 through PCR of CSF and prompt treatment with acyclovir may prevent devastating neurological sequelae.

(C) 2019 The Author(s)

Published by S. Karger AG, Basel

\section{Introduction}

Herpes simplex virus type 2 (HSV-2) is responsible for the majority of genital herpes infections. Approximately 45 million people in the United States have genital herpes, with an estimated one million new infections annually [1]. Similar to HSV type 1 (HSV-1) and varicella zoster virus (VZV), the HSV-2 virus establishes latency in the peripheral sensory ganglia and persists in the host for a lifetime $[1,2]$. These infections often reactivate and lead to significant morbidity and mortality $[2,3]$. Typically transmitted during childhood via orolabial mucocutaneous surfaces, HSV-1 primarily causes herpes labialis, encephalitis, and corneal blindness $[2,4]$. Primary HSV-2 infection is frequently responsible for meningoencephalitis in neonates acquired through vaginal delivery, whereas reactivated HSV-2 is usually associated with meningitis in adults $[2,4,5]$. Radiculomyelitis is rarely encountered with HSV-2, especially in immunocompetent individuals [1].

Conversion disorder, also known as functional neurological symptom disorder, is defined as a psychiatric illness in which signs and symptoms affecting voluntary motor or sensory function cannot be explained by a neurological or general medical condition [6, 7]. As a potentially reversible source of disability, these disorders are often misdiagnosed or correctly diagnosed after extended delays [7].

Herein, we report a unique case of a patient with HSV-2 presenting as lower extremity pain and weakness. We examine her neurological signs and symptoms as well as differential diagnosis in the context of her psychiatric history and discuss the medical management and long-term prognosis of $\mathrm{HSV}-2$ radiculomyelitis.

\section{Case Description}

A 44-year-old woman (height: 5'4" [1.6 m]; weight: $185 \mathrm{lbs}$ [83.9 kg]; body mass index: 31.75) presented to the Emergency Department with complaints of severe low back pain and bilateral lower extremity pain and weakness. She stated that the symptoms initiated one day earlier with muscle cramping of the bilateral posterior thighs and had gradually progressed to an inability to walk or lift her legs. The patient initially attributed her symptoms to dehydration as she had worked several 12-h shifts in a row as a registered nurse. She reported some urinary hesitancy although denied urinary or bowel incontinence, any sensory deficits of the lower extremities, or severe headaches. The patient mentioned several stressors, including marriage in the next week to a man to whom she had been previously married, working overtime, minimal sleeping at night, and long commute to work. Past medical history was significant for anxiety, depression, and migraines. The patient denied a history of sexually 


\section{Case Reports in Neurology}

Case Rep Neurol 2019;11:117-123

DOI: $10.1159 / 000499701$

Shields and Alsorogi: Conversion Disorder

transmitted diseases. Two years earlier, the patient was hospitalized for $24 \mathrm{~h}$ following complaints of right hemiparesis and chest pain. She was diagnosed with conversion disorder due to a psychological stressor.

The physical examination revealed $1 / 5$ power in the lower extremities bilaterally, with distal strength greater than proximal. Sensation, deep tendon reflexes, and muscle tone of the lower extremities were normal. There was an absence of atrophy or hypertrophy of the muscles, rigidity or spasticity, and tremors or abnormal movements. Serial cervical, thoracic, and lumbar MRI scans with and without gadolinium contrast demonstrated no significant stenosis, neural compression, or other etiologies of her symptoms. The brain MRI with and without gadolinium contrast was normal. The initial impression was conversion disorder due to the patient's multiple work and familial stressors. She was hospitalized and treated with methylprednisolone and hydromorphone.

A lumbar puncture and a CSF pathogen panel were performed, the latter utilizing a multiplex nested PCR followed by a melting analysis to detect nucleic acid sequences. The sensitivity was greater than $95 \%$, and the specificity was greater than $99.6 \%$. The panel detected HSV-2 and was negative for crypococcal antigen. The following findings were also observed in the CSF: glucose $90 \mathrm{mg} / \mathrm{dL}(40-70 \mathrm{mg} / \mathrm{dL})$, protein $55 \mathrm{mg} / \mathrm{dL}(12-60 \mathrm{mg} / \mathrm{dL}), \mathrm{WBC} 434 / \mu \mathrm{L}$ $(0-5 / \mu \mathrm{L}), \mathrm{RBC} 9 / \mu \mathrm{L}(0-2 / \mu \mathrm{L})$, lymphocytes $94 \%$ (40-80\%), and monocytes 6\% (15-45\%). The culture CSF/Gram stain revealed rare WBCs and no organisms, and the HIV-1 and -2 antibody/antigen combination was nonreactive. Borrelia burgdorferi antibodies (Lyme disease), syphilis total antibodies, hepatitis B core antibodies and surface antigens, Chlamydia trachomatis, and Neisseria gonorrhoeae were all negative. The patient was diagnosed with radiculomyelitis due to HSV-2 marked by back pain and weakness of the lower extremities with evidence of lymphocytic pleocytosis. She initiated treatment with acyclovir $650 \mathrm{mg}$ infused every $8 \mathrm{~h}$ for 3 weeks and was subsequently prescribed valacyclovir.

The patient had attained no improvement of her lower extremity pain when evaluated by a neurologist 5 and 8 months following the start of her symptoms. Additionally, CSF findings demonstrated a WBC count $6 / \mu \mathrm{L}$, protein $33 \mathrm{mg} / \mathrm{dL}$, and glucose $57 \mathrm{mg} / \mathrm{dL} 5$ months after her initial lumbar puncture, respectively. There were greater than 4 oligoclonal bands detected in the CSF with no corresponding bands detected in the serum, and the IgG index was 0.9 [0.30.7]. These positive findings indicated an inflammatory process. The aquaporin 4 (AQP4) receptor antibody was negative. The patient was evaluated by a neurologist 17 months after her symptoms initiated, at which time she had significant improvement in the range of motion strength in her lower extremities. Her minimal residual pain was controlled with gabapentin, baclofen, and alprazolam.

\section{Discussion}

The mixed clinical and radiological picture with accompanying psychiatric and CSF findings warrants a thorough examination of the differential diagnosis in our case, specifically, conversion disorder, Guillain-Barré syndrome (GBS), radiculomyelitis, and neuromyelitis optica (NMO) (Table 1). Sigmund Freud coined the term conversion disorder and hypothesized that symptoms not explained by organic diseases reflect unconscious conflict [6]. Stressors such as trauma or psychological distress present as a physical deficit, although there is no 


\section{Case Reports in Neurology}

Case Rep Neurol 2019;11:117-123

DOI: $10.1159 / 000499701$

2019 The Author(s). Published by S. Karger AG, Base www.karger.com/crn

Shields and Alsorogi: Herpes Simplex Virus Type 2 Radiculomyelitis Disguised as Conversion Disorder

underlying physical cause of the symptoms nor can the affected individuals control the symptoms [6]. Common conversion symptoms include blindness, paralysis, dystonia, psychogenic nonepileptic seizures, anesthesia, swallowing difficulties, motor tics, difficulty walking, hallucinations, anesthesia, and dementia.

GBS, or acute inflammatory demyelinating polyradiculoneuropathy, is marked by acute ascending motor paresis, diminished or absent deep tendon reflexes, minimal objective sensory loss, electrophysiologic evidence of a demyelinating neuropathy, and CSF albumniocytologic dissociation [8]. GBS may result from an immune response to a preceding infection, most commonly Campylobacter jejuni infection, cytomegalovirus (CMV), Epstein-Barr virus (EPV), Haemophilus influenzae, and Mycoplasma pneumonia [9]. HSV IgM-specific antibodies have rarely been detected in GBS [10].

HSV-2 infection is rarely associated with radiculomyelitis, especially in patients who are immunocompetent [1,3]. HSV-2 radiculomyelitis affects the lumbar or sacral nerve roots and may cause radicular pain, paresthesia, urinary retention, constipation, anogenital discomfort, and leg weakness $[11,12]$. Loss of deep tendon reflexes of the lower extremities may also be observed [12]. The differential diagnosis of radiculomyelitis includes HSV-1, HSV-2, CMV, EBV, VZV, and enteric cytopathogenic human orphan virus [11]. The CSF analysis, PCR, and MRI confirm the diagnosis in cases of HSV-2 lumbosacral radiculomyelitis $[4,11,13]$. The CSF usually reveals a lymphocytic pleocytosis and a minor elevation of CSF protein. The most sensitive and specific method for detecting HSV-2 DNA is the rapid real-time polymerase chain reaction assay in the CSF and serum. MRI findings include sacral root or lower spinal cord edema with enlargement and hyperintensity on T2-weighted images as well as contrast enhancement in acute infection. However, the MRI may be negative. Interestingly, several viral conditions known to cause radiculomyelitis may demonstrate normal spinal MRIs, including HSV1, HSV-2, VZV, CMV, adenovirus, enterovirus, coxsackie B virus, and herpes virus 6 [14].

Acyclovir is the preferred treatment in cases of confirmed HSV-2 radiculomyelitis. Vidarabine and corticosteroids have also been shown to hasten improvement $[3,11]$. Close monitoring is recommended following an episode of herpetic meningitis and radiculomyelitis, as it has been reported that approximately $30 \%$ of patients experience a recurrence of symptoms within 1 year [5]. Furthermore, in Suarez-Calvet and colleagues' review of 13 patients with lumbar polyradiculopathy caused by HSV-1 or HSV-2, 8 (61.5\%) patients had complete or partial recovery, 4 (30.8\%) had no improvement, and 1 (7.7\%) died [12]. The WBCs of the patient in our case dropped significantly from 434 to $6 / \mu \mathrm{L}$ over a 6 -week period following her HSV-2 confirmation, diagnosis of radiculomyelitis, and initiation of acyclovir, indicating that the inflammatory processes were resolving. She attained no improvement of her symptoms within 8 months; however, she reported decreased pain and improved strength of the lower extremities by 17 months.

Described by Elsberg in 1931, Elsberg syndrome is characterized by a cauda equina syndrome and lower thoracic myelitis [11]. It is an infectious syndrome consisting of acute or subacute bilateral lumbosacral radiculitis, myelitis confined to the lower spinal cord, and acute urinary retention. It is commonly caused by a reactivation of an HSV-2 infection in the spinal ganglia. While Elsberg syndrome is often a self-limiting syndrome, it may lead to ascending necrotizing myelitis with a fatal outcome within weeks in immunosuppressed patients [11]. 
NMO, or Devic's disease, is a rare inflammatory and demyelinating autoimmune disorder of the CNS characterized by recurrent occurrences of optic neuritis and longitudinally extensive transverse myelitis [15]. The presence of NMO-IgG/AQP4 antibodies in serum supports the diagnosis of NMO.

Due to our patient's history of a documented conversion disorder 2 years earlier, this diagnosis was initially considered, and the patient was only treated with corticosteroids and hydromorphone. The diagnosis of GBS was contemplated; however, the patient's preserved deep tendon reflexes in the lower extremities were not consistent with this demyelinating disease. Upon the confirmation of HSV-2 by PCR of the CSF, the diagnosis of radiculomyelitis was validated. Our patient did not display the classic findings associated with radiculomyelitis since her deep tendon reflexes were intact and her spinal MRI was normal. These findings are not observed in all cases of HSV-2 radiculomyelitis $[12,14]$. We believe that our case represents the rare Elsberg syndrome, replete with acute bilateral radiculomyelitis and urinary abnormalities. NMO was excluded as the AQP4 receptor antibody was negative.

In conclusion, neurologists should consider HSV-2 when presented with a patient displaying radiculomyelitis, especially when the psychiatric picture of conversion disorder is convincing. Although the spinal MRI may be negative, it is important to consider an infectious process which may be treatable. An early and accurate diagnosis of HSV-2 coupled with prompt acyclovir administration is imperative to mitigate the devastating neurological complications that may ensue.

\section{Acknowledgment}

We acknowledge Norton Healthcare for their continued support.

\section{Statement of Ethics}

The University of Louisville Institutional Review Board has determined that our project does not meet the "Common Rule" definition of human subjects' research and does not require IRB review. The Institutional Review Board number is 19.0140. The patient described in this article provided approval and informed consent.

\section{Disclosure Statement}

The authors have no conflicts of interest to declare.

\section{Funding Sources}

None. 


\section{References}

1 Berger JR, Houff S. Neurological complications of herpes simplex virus type 2 infection. Arch Neurol. 2008 May;65(5):596-600.

2 Steiner I, Benninger F. Update on herpes virus infections of the nervous system. Curr Neurol Neurosci Rep. 2013 Dec;13(12):414.

3 Nardone R, Versace V, Brigo F, Tezzon F, Zuccoli G, Pikija S, et al. Herpes simplex virus type 2 myelitis: case report and review of the literature. Front Neurol. 2017 May;8:199.

4 Benjamin MM, Gummelt KL, Zaki R, Afzal A, Sloan L, Shamim S. Herpes simplex virus meningitis complicated by ascending paralysis. Proc Bayl Univ Med Cent. 2013 Jul;26(3):265-7.

5 Aurelius E, Forsgren M, Gille E, Sköldenberg B. Neurologic morbidity after herpes simplex virus type 2 meningitis: a retrospective study of 40 patients. Scand J Infect Dis. 2002;34(4):278-83.

6 Ali S, Jabeen S, Pate RJ, Shahid M, Chinala S, Nathani M, et al. Conversion disorder- mind versus body: A review. Innov Clin Neurosci. 2015 May-Jun;12(5-6):27-33.

7 Espay AJ, Aybek S, Carson A, Edwards MJ, Goldstein LH, Hallett M, et al. Current concepts in diagnosis and treatment of functional neurological disorders. JAMA Neurol. 2018 Sep;75(9):1132-41.

8 Oh SJ, LaGanke C, Claussen GC. Sensory Guillain-Barré syndrome. Neurology. 2001 Jan;56(1):82-6.

9 Tam CC, O’Brien SJ, Petersen I, Islam A, Hayward A, Rodrigues LC. Guillain-Barré syndrome and preceding infection with campylobacter, influenza and Epstein-Barr virus in the general practice research database. PLoS One. 2007 Apr;2(4):e344.

10 Gerken G, Trautmann F, Köhler H, Falke D, Bohl J, Nix W, et al. Rare association of herpes simplex virus IgMspecific antibodies and Guillain-Barré syndrome successfully treated with plasma exchange and immunosuppression. Klin Wochenschr. 1985 May;63(10):468-74.

11 Eberhardt 0, Küker W, Dichgans J, Weller M. HSV-2 sacral radiculitis (Elsberg syndrome). Neurology. 2004 Aug;63(4):758-9.

12 Suarez-Calvet M, Rojas-Garcia R, Querol L, Sarmiento LM, Domingo P. Polyradiculoneuropathy associated to human herpesvirus 2 in an HIV-1-infected patient (Elsberg syndrome): case report and literature review. Sex Transm Dis. 2010 Feb;37(2):123-5.

13 Ellie E, Rozenberg F, Dousset V, Beylot-Barry M. Herpes simplex virus type 2 ascending myeloradiculitis: MRI findings and rapid diagnosis by the polymerase chain method. J Neurol Neurosurg Psychiatry. 1994 Jul;57(7):869-70.

14 Wong SH, Boggild M, Enevoldson TP, Fletcher NA. Myelopathy but normal MRI: where next? Pract Neurol. 2008 Apr;8(2):90-102.

15 Sellner J, Boggild M, Clanet M, Hintzen RQ, Illes Z, Montalban X, et al. EFNS guidelines on diagnosis and management of neuromyelitis optica. Eur J Neurol. 2010 Aug;17(8):1019-32. 


\section{Case Reports in Neurology}

Case Rep Neurol 2019;11:117-123

DOI: $10.1159 / 000499701$

(C) 2019 The Author(s). Published by S. Karger AG, Base www.karger.com/crn

Shields and Alsorogi: Herpes Simplex Virus Type 2 Radiculomyelitis Disguised as Conversion Disorder

Table 1. Differential diagnosis, investigation, and treatment in the present case

Differential diagnosis

Conversion disorder

Guillain-Barré syndrome

Lumbar radiculomyelitis

Neuromyelitis optica

Investigation

History and physical examination

Lumbar puncture with CSF pathogen panel and polymerase chain reaction

Brain, cervical, thoracic, and lumbar MRI scans with and without gadolinium contrast

HIV-1 and -2

Borrelia burgdorferi (Lyme disease)

Syphilis

Hepatitis B

Chlamydia trachomatis

Neisseria gonorrhoeae

Oligoclonal bands

IgG index

Aquaporin 4

Treatment

Methylprednisolone

Hydromorphone

Acyclovir

Valacyclovir

Gabapentin

Baclofen

Alprazolam 